\title{
A study on the Model of 3D-coordinate Measuring Machine Error Compensation Based on Finite Element Simulation
}

\author{
Jizhu Liu, Shuanghui Hao, Minghui Hao and Baoyu Song \\ School of Mechatronic Engineering, Harbin Institute of Technology, Harbin, China, liu_jizhu@163.com
}

\begin{abstract}
:
Additional inertial force will cause the deformation of some parts when 3D-coordinate Measuring Machine (CMM) runs quickly. It will result in the difference between real results and reading of raster sensor, that is, dynamic error will be produced. This paper presents the theoretical model of CMM dynamic error compensation.The Z-direction deformation of crossbeam is analyzed based on Ansys, one kind of finite element simulation software, when slide carriage is in different position under three different load conditions (accelerating,uniform speed, decelerating). Dynamic error compensation model is acquired under any kind of load according to the analysis results, which can improve the measuring accuracy of CMM greatly.
\end{abstract}

Keywords: CMM, Error Compensation, Finite Element Analysis, Dynamic Errors

\section{Introduction}

With the development of modern mechanical manufacturing, fast movement and high precision is required for 3D-coordinate Measuring Machine (CMM). In order to improve the accuracy of CMM, more and more researchers are attracted by dynamic error compensation. The dynamic error of CMM is caused by the offset of moving parts around the joint with guide way and their own deformation. The stiffness of guide way and moving parts is limited. So the offset and deformation occurs when CMM accelerates or decelerate, because of the additional inertial force. It will results in the difference between real results and reading of raster sensor, that is, dynamic error will be produced. Some error compensation models are built by many researchers, which are required according to much experimentation. This paper presents a theoretic dynamic error compensation model in Z-direction, which can reduce measurement error extraordinary.

\section{Theoretic Analysis}

The gantry CMM $(400 \mathrm{~mm} \times 400 \mathrm{~mm} \times 400 \mathrm{~mm})$ designed by this paper is shown in Fig.1.

The crossbeam glides in Y-direction. The slide carriage moves in $\mathrm{X}$-direction, and the feeler pin runs in Z-direction. In the process of fast movement, additional inertial force is produced when slide carriage and

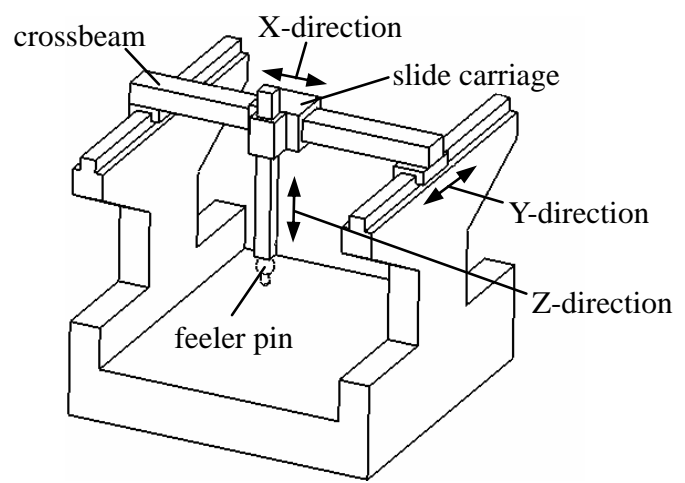

Fig.1 Simplified schematic diagram of CMM the crossbeam glides in Y-direction. The slide carriage moves in X-direction, and the feeler pin runs in Z-direction. In the process of fast movement, additional inertial force is produced when slide carriage and Z-direction spindle accelerates or decelerates. So the crossbeam will bend and deflect. The load condition is shown in Fig.2 in Z-direction.

$p$ is the sum of all forces in Z-direction including additional inertial force, gravity of Z-direction spindle and slide carriage. $\mathrm{q}$ is the load of unit length. The crossbeam will bend round Y-direction and deflect round the joint with guide way, if Z-direction spindle changes its speed when slide carriage moves in X-direction. The following relational expression can be acquired 


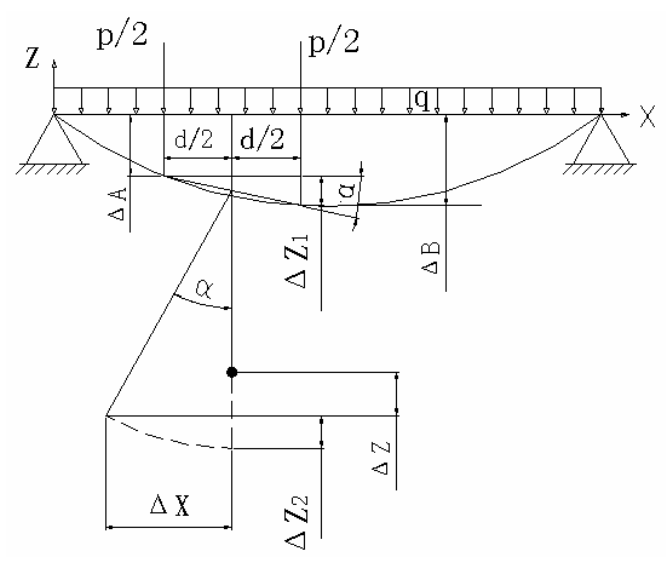

Fig.2 The deformation schematic diagram of crossbeam under load

according to Fig.2.

$$
\begin{gathered}
\Delta Z=\Delta A+\Delta Z_{1}-\Delta Z_{2} \\
\Delta Z_{2}=l(1-\cos \alpha) \\
\Delta Z_{1}=\frac{1}{2}(\Delta B-\Delta A) \\
\Delta Z=\Delta A+\frac{1}{2}(\Delta B-\Delta A)-l(1-\cos \alpha)
\end{gathered}
$$

If $\alpha \rightarrow 0$, then $\sin \alpha \sim \alpha \sim \tan \alpha$. So the following can be deduced.

$$
\begin{aligned}
& 1-\cos \alpha \sim \frac{1}{2} \alpha^{2} \\
& \Delta Z \approx \Delta A+\frac{1}{2}(\Delta B-\Delta A)-\frac{1}{2} \alpha^{2} l
\end{aligned}
$$

The offset of Z-direction spindle along $\mathrm{X}$-direction is given in Eq.7.

$$
\Delta X_{l}=l \sin \alpha=l \tan \alpha
$$

The offset error caused by deformation of crossbeam is relative to the position of Z-direction spindle, that is, $l$. If $l=1 \mathrm{~m}$, then

$$
\Delta X \approx \tan \alpha=\frac{|\Delta B-\Delta A|}{|A B|}
$$

\section{Finite Element Analysis}

The crossbeam is made of granite. The density is $3 \mathrm{~g} / \mathrm{cm}^{3}$. The elastic modulus is 120GPa, and the Poisson ratio is 0.3. The dimensions is shown in Fig.3. The model is meshed using SOLID95(3D elements) of ANSYS, which has 20 nodes. The load step is applied every $20 \mathrm{~mm}$, in order to simulate the load condition of crossbeam. Three different kind of load conditions are given in this paper. $\mathrm{p}$ is $500 \mathrm{~N}$ when Z-direction spindle accelerates. $\mathrm{p}$ is $450 \mathrm{~N}$ when spindle moves at uniform speed. $\mathrm{p}$ is $400 \mathrm{~N}$ when spindle decelerates. The speed and position is controlled by the motor. p and q can be calculated according if acceleration is certain. Then the offset error of feeler pin can be calculated according to Eq.6 and Eq.8 in different position under the three load condition. The error of the feeler pin in Z-direction and in X-direction is shown in Fig.4 and Fig.5.

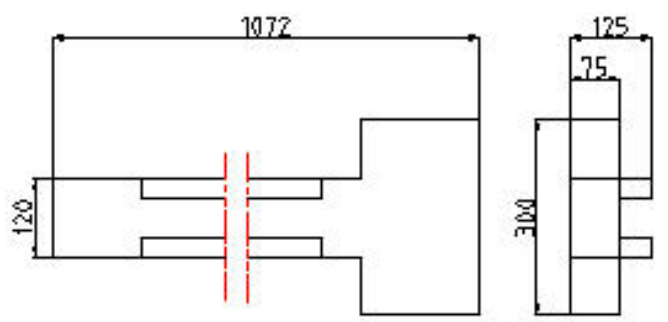

Fig.3 Simplified schematic diagram of crossbeam structure

\section{Error Modeling}

The error in Z-direction and in X-direction is produced by the deformation of crossbeam when Z-direction spindle accelerates. There is one point in crossbeam (the abscissa is $X_{m}$ ). The offset error of the point in X-direction is zero. So the mathematical model of the offset error can be built in two parts. Firstly, the point must be found in which the offset error in Z-direction is maximal. The point is dividing point of error compensation functions. The error compensation functions in Z-direction and in X-direction can be built in terms of Lagrange Interpolation. These functions are given in Eq.9 Eq.11.

In a similar way, the error compensation models can be acquired when Z-direction spindle moves at uniform speed, as shown in Eq.12 Eq.14. The model is shown in Eq.15 Eq.17 when the spindle decelerates.

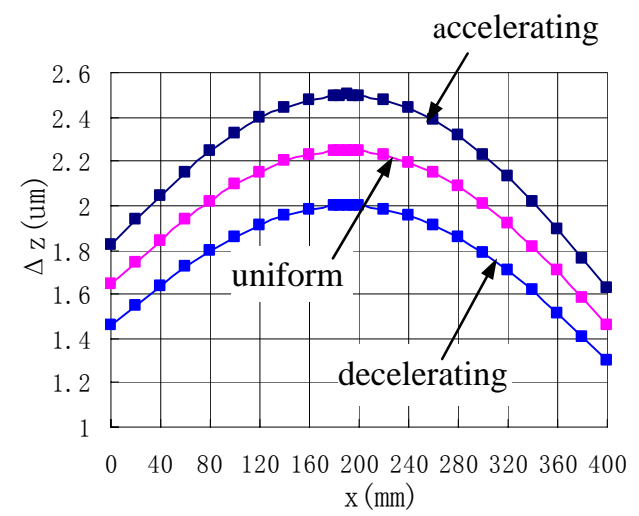

Fig.4 Error curve in Z-direction 


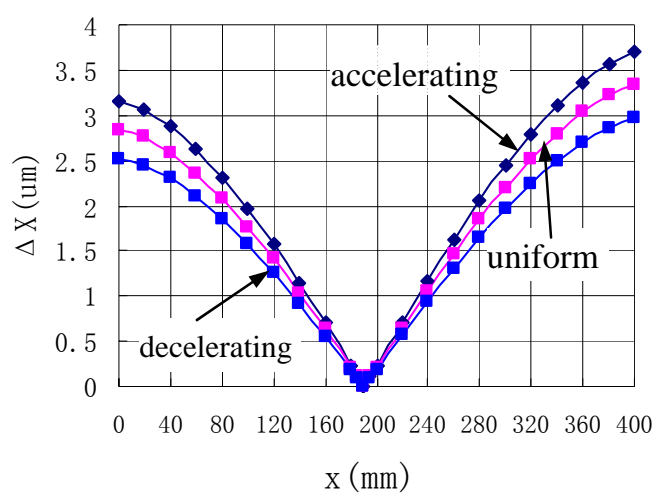

Fig.5 Error curve in X-direction

The error compensation model is given in the Eq.9 Eq.11 when Z-direction spindle accelerates.

$$
\begin{gathered}
\Delta Z_{1}^{\prime}(x)=\left(-0.204925 x^{2}+77.219 x\right. \\
+17707) \times 10^{-4} \mu \mathrm{m} \\
\Delta X_{1}^{\prime}(x)=\left(-0.5074072 x^{2}-81.55555 x\right. \\
+32818.52) \times 10^{-4} \mu \mathrm{m} \\
\left(0 \leq x \leq X_{M}\right) \\
\Delta X_{1}^{\prime \prime}(x)=\left(-0.4755953 x^{2}+466.0441 x\right. \\
-72433.03) \times 10^{-10} \mu m \\
\left(X_{M} \leq x \leq 400 \mathrm{~mm}\right)
\end{gathered}
$$

The error compensation model is given in the Eq.12 Eq.14 when Z-direction spindle moves at uniform speed.

$$
\begin{gathered}
\Delta Z_{2}^{\prime}(x)=\left(-0.163940887 x^{2}+61.77635 x\right. \\
+14165.75) \times 10^{-4} \mu m \\
\Delta X_{2}^{\prime}(x)=\left(-0.40601825 x^{2}-65.194445 x\right. \\
+26251.847) \times 10^{-4} \mu m \\
\left(0 \leq x \leq X_{M}\right) \\
\Delta X_{2}^{\prime \prime}(x)=\left(-0.38115 x^{2}+373.21043 x\right. \\
-57997.4752) \times 10^{-4} \mu m \\
\left(X_{M} \leq x \leq 400 \mathrm{~mm}\right)
\end{gathered}
$$

The error compensation model is given in the Eq.15 Eq.17 when Z-direction spindle accelerates.

$$
\begin{gathered}
\Delta Z_{3}^{\prime}(x)=\left(-0.1844178 x^{2}+69.49055573 x\right. \\
+15936.94624) \times 10^{-4} \mu m \\
\Delta X_{3}^{\prime}(x)=\left(-0.45509275 x^{2}-73.694415 x\right. \\
+29548.147) \times 10^{-4} \mu m \\
\left(0 \leq x \leq X_{M}\right) \\
\Delta X_{3}^{\prime \prime}(x)=\left(-0.429563664 x^{2}+420.2977167 x\right. \\
-65302.39413) \times 10^{-4} \mu m \\
\left(X_{M} \leq x \leq 400 \mathrm{~mm}\right)
\end{gathered}
$$

\section{Error Evaluation}

The results of error compensation model are compared with finite element simulation. The residual error curves in Z-direction and in X-direction are shown in Fig.6 and Fig.7.

It can be concluded that the measuring error is decreased greatly from Fig.6 and Fig.7.

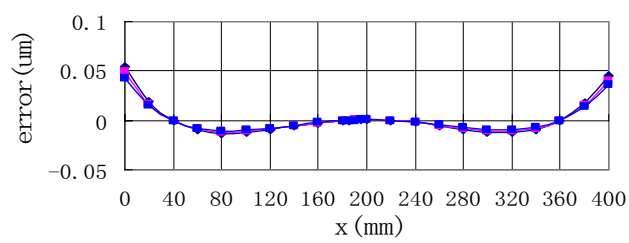

Fig.6 The residual error curves in Z-direction

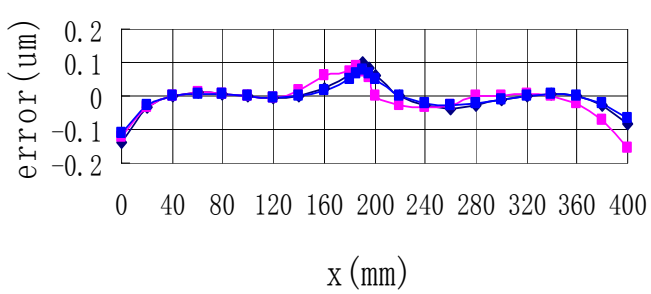

Fig.7 The residual error curves in X-direction

\section{Universal mathematical model}

A rule is found through much analysis. The ratio of the corresponding coefficients of error compensation functions is same with the ratio of applied force under any two kinds of load condition, which accords with Hooke Law. So according to one certain error compensation function, any other functions can be obtained. The universal error compensation function of CMM in Z-direction is given in Eq.18, and the functions in X-direction are shown in Eq.19 and Eq.20. 


$$
\Delta Z^{\prime}(x)=\frac{P\left(-0.204925 x^{2}+77.219 x+17707\right)}{500} \times 10^{-4} \mu m
$$

$\Delta X_{l}^{\prime}(x)=\frac{P\left(-0.5074072 x^{2}-81.55555 x+3281852\right)}{500} \cdot l \times 10^{-4} \mu \mathrm{m}$

$$
\left(0 \leq x \leq X_{M}\right)
$$

$$
\begin{array}{r}
\Delta X_{l}^{\prime \prime}(x)=\frac{P\left(-0.4755953 x^{2}+466.0441 x-72433.03\right)}{500} \cdot l \times 10^{-4} \mu m \\
\left(X_{M} \leq x \leq 400 \mathrm{~mm}\right)
\end{array}
$$

Where, $\quad l--$ the displacement of feeler pin in Z-direction.

\section{Conclusion}

This paper simulates the deformation of crossbeam in Z-direction under three different load conditions (accelerating, uniform speed, decelerating). Three error compensation models are presented. An universal mathematical model under any kind of load conditions is deduced based on the former three models. The calculating results of the models are compared with simulation results from Ansys. The comparison verifies the validity of the theoretical model of CMM dynamic error compensation, so the measurement error can be reduced greatly using the model in this paper.

\section{References}

[1] Dong Chensong, Zhang Guoxiong, Mu Yuhai,1998, Assessing the Dynamic Errors of Coordinate Measuring, China Mechanical Engineering, Vol.9, no.7,pp ,34-36.

[2] Y. H. Mu and B. K. A. Ngoi, 1999, Dynamic Error Compensation of coordinate Measuring Machines for High-speed Measurement, The International Journal of Advanced Manufacturing Technology, no.15, pp.810-840.

[3] Liu Peng, Lin Shuwen, 2001, Error Analysis and Compensation of Non-rigid Errors of CMM, Tool Engineering, vol.35,no.10,pp,32-34.

[4] J. F. C. De Nijs.1988,Modeling of a Coordinate Measuring Machine for Analysis of Its Dynamic Behavior, Annals of the CIRP ,vol,37,pp.507 510.

[5] Fei Yetai, Zhao Jing, Wang Hongtao, Ma Xiushui, 2004, A Review of Research on Dynamic Errors of Coordinate Measuring Machines, Chinese Journal of Scientific Insrument, vol.25, no.4, pp.773-776. 